\title{
A lonely business: reflections on the wellbeing and morale of dental teams
}

\author{
Natasha Hayer ${ }^{1}$ and Hoda S. Wassif*2
}

\section{Key points}

Explores the subject of team morale in the dental practice.
Offers a reflection on the dental team dynamic and how it may influence patient care.
Proposes a strategy for change to enhance team interactions.

\begin{abstract}
With the evolution of humanising patient-dentist interaction within healthcare has come an equal need to humanise staff interaction in order to optimise patient care. There is growing evidence to support the need to invest in enhancing team morale, given that its impact, when low, results in reduced quality of patient care. This paper endeavours to reflect on dental teams' wellbeing and morale and propose a strategy for change to enhance these aspects of team interactions. The theme throughout is to focus on staff wellbeing, in order to initiate a change in work ethic which should in turn initiate a positive work environment. The two strategies discussed are leadership changes conducive to a servant leadership style and a mentorship/buddy programme focusing on reducing stress among dental professionals. Both strategies have their merits and faults, but the purpose of the paper is to prompt food for thought as to how to raise team morale in dental practice.
\end{abstract}

\section{Introduction}

It is without doubt that the key factors which pertain to a productive and advancing team are human-oriented. That is to say, like the Hawthorne effect and following experiments, ${ }^{1}$ it has been proven time and again that it is not always the surroundings or external influences which enhance a team but the attention to individuals that helps to contribute to a flourishing team. With much emphasis placed on 'humanising' patient care by developing trust between patient and doctor, developing trust within healthcare teams and 'humanising' their interactions, seems at times to be undervalued. Team morale has long been acknowledged as integral to a happy working environment, leading to enhanced performance within

'Dental Surgery, 180 Northridge Way, Hemel Hempstead, UK; ${ }^{2}$ University of Bedfordshire, Putteridge Bury Campus, Hitchin Road, Luton, UK.

Correspondence to: Hoda Wassif

Email: hoda.wassif@beds.ac.uk

Refereed Paper.

Accepted 21 December 2018

DOI:10.1038/s41415-019-0205-y an organisation. Its importance is palpable when team morale is low, particularly when the organisation in question primarily deals with the public. The literature claims that low team morale can impact quality of patient care, particularly where low morale leads to burnout, a term coined to explain the mental, physical and emotional exhaustion experienced by employees working in emotionally demanding situations. ${ }^{2,3}$

'You're in a career where nobody wants to see you and you're the last place they want to come back to and it's depressing. ${ }^{4}$ This is the view of one dental practitioner and in reality, low morale matters as it impacts the dental profession as a whole. Research by the King's Fund ${ }^{5}$ discussed in depth the importance of morale and motivation within the NHS. It highlighted that good morale and motivation have positive effects on patient care and acknowledged that measuring morale is difficult, often assessed 'anecdotally, for example, through comments from staff or "picking up on a feeling". ${ }^{5}$ Others highlighted how staff morale directly influences patient experience and care, ${ }^{5}$ with positive emotions generated by staff resulting in positive responses from patients or so-called 'emotional contagion. $^{6}$

However, surface acting emotion, which lacks authenticity, is shown to increase stress levels and reduce job satisfaction and therefore the emotion must be deep acting and have authenticity. The biggest foreseeable challenge, therefore, is encouraging teams to genuinely generate positive emotions. Striving to keep every team member happy is likely to be unrealistic, but what is being proposed here is how to instil change in the way staff thinks, so they can generate their own motivations and happiness.

\section{The working environment and feeling valued}

The King's Fund research review outlined some common factors which influence morale within healthcare teams. ${ }^{5}$ Interaction is predominantly between dentist and nurse through both a social and professional means, the latter being limited, thus leading to a feeling of professional isolation but conversely an alternative albeit dissimilar social outlet. The isolated dentist pattern tends to transcend past 
the dental practice into the wider community as well. Again, there is no real need to communicate with other dentists outside the practice, as there is no wider network or team which require it for a practitioner to deliver good clinical practice.

Despite the claim of promoting 'learning culture' within the NHS, ${ }^{5}$ staff members are often left feeling that they were blamed for errors which changed public perception of them for the worse. On a personal level, staff felt more valued if their superiors made a genuine effort to get to know them or listened to their views and ideas. The same review discussed differences between peer groups which may have more qualifications than another, resulting in an egocentric interaction rather than a nurturing one. The danger being those who wish to train further, due to lack of support, either abandon the career altogether or remain stagnant in a zone of complacency, thus breeding further low morale. This is more relevant to dental teams as they also face more isolation due to the nature of their daily clinical work.

There are few groups which encourage membership to be part of a community; however, subscription charges are generally high and often act as a barrier, for example to those in part-time employment. The practice of defensive dentistry has also been antagonistic to team care and this can be clearly seen in general practice where dentists tend to be focused on covering themselves rather than focusing on a team approach to dentistry. Defensive dentistry has been cited frequently as lowering morale and increasing stress for dentists. ${ }^{7}$

Poor leadership is often cited as one of the most frequent reasons for staff low morale. ${ }^{8}$ More specifically, poor interpersonal connections between staff and leaders, distrust of leaders, and inflexible working conditions led to poor morale. Research suggests to alter leadership strategy towards a servant leadership, where the focus is centred around trust and empowerment. ${ }^{9}$ It is argued that servant leadership can assist in providing high-quality health care. ${ }^{10,11}$ This would be a hard change in ethos for many dental practices where there is a mixture of transactional and adaptive leadership strategies. That is to say, a good job has been traditionally rewarded monetarily and problems 'solved' using a reflective and collaborative approach. Both approaches work incredibly well, though only for a very short amount of time as the pattern becomes cyclic.

\section{A call for change}

Changing leadership strategy and encouraging staff mental wellbeing need to work in synchronisation in order to ensure positive patient experience that relies, in turn, on a team which is well supported. The first strategy for change involves a shift toward servant leadership, utilising its characteristics, where the focus for reward or happiness within the working environment arises from developing and supporting individuals. The fundamental difference between the transformational style of leadership and the servant style is that, with the latter, the needs of the people involved are prioritised over the needs of the organisation. ${ }^{12}$ The hope is to instil an ethos of trust and support, where it can also be reciprocated back to the leader, thus creating a feeling of unity and enhanced performance within the team. ${ }^{13}$

\section{Leadership strategy}

Changing leadership style from a traditional, transformational one to the servant type is complex, as it means changing the approach the current leader takes regarding many issues in practice. As previously mentioned, a servant leader is required to show the ability to empower and gain trust, and also display empathy. ${ }^{8}$ These qualities may be unnatural to some and, therefore, introducing these to a leader who is used to implementing a different style may prove challenging. Moreover, a leader who is also a stakeholder in the business creates an immediate conflict of interest, particularly when prioritising the needs of staff before the organisation. Creating a role of 'sub-leader', who does not have this conflict of interest, could be one way of ensuring a focus on serving the wider team and ensuring their empowerment. ${ }^{14}$ Therefore, you would have the transformational leader and servant leader working alongside each other but each maintaining a different focus; one on the business and the other on the staff.

The most glaringly obvious challenge with this setup is when the needs of the business and those of its staff conflict. There are infinite scenarios when this could occur, and it would be up to both leaders to develop a plan to handle each scenario with an intention to minimise staff unhappiness and negative impact on the business. What may have to be adopted as the bottom line when making this compromise is that, just like in clinical scenarios, the patient's interest is what ought to come first. In other words, if staff unhappiness results in patient care suffering, patient care would take precedence.

One may question why someone would want to be a servant leader. If the emphasis is on enhancing team morale by developing individual team members, what is the motivation for the leader to do this? However, it is not so much the need for less power from the leader but a different way of dealing with that power. 'Helping power motivation' has been cited as the driving force in servant leadership. ${ }^{15}$ That is, a desire for power with the purpose of helping and/or caring for others. This almost parallels with a Kantian view on leadership, ${ }^{15}$ which suggests a responsibility of the leader to instil autonomy in its followers and encourage thinking for themselves. Indeed, this highly benevolent view of leadership appears to be idealistic to achieve. Does such a leader exist? Given that servant leadership entails using persuasion over power to form relationships with followers, can this persuasion become biased or coercive? The concept of placing importance on an individual within an organisation has the power to generate an ethos of the individual having an innate desire to want to be the best they can be, so it appears nonsensical not to use this concept explicitly to create an atmosphere which can impact health care in a positive way. However, clear challenges can be seen with regards to the practicalities of this style of leadership. The question would be, can elements of this style of leadership be employed in dental practice in order to generate a subtle enhancement of morale?

\section{Mentorship}

The notion of Kantian style of helping power motivation ${ }^{15}$ parallels with the second strategy for enhancing team morale to focus on staff mental wellbeing, through the development of buddy/mentorship programmes within dental practice. There is a growing trend to deliver a sense of autonomy to those being mentored by facilitating their learning, ${ }^{16}$ therefore a mentor need not be necessarily someone senior but one who has the emotional intelligence and psychological maturity to aid professional growth. This marks a shift from a mentor having an advisory role to one that works alongside their mentee, forming a symbiotic relationship. The mentor can share vast experience and the mentee can take on the role of educating the mentor in new methods or advancements with certain areas of expertise. This collaborative, symbiotic approach removes the traditional hierarchical setup and creates a more defined 
sense of team; whereby every member of varying experience is learning, not just those on the base of the hierarchy.

The traditional mentorship setup should not only include discussing difficult cases but also allow the opportunity for mentees to 'get things off their chest', with a view to working collaboratively, thus optimising patient care. As well as clinical scenarios and team approaches to patient treatment planning, incorporating the emotional impact of patient care into the discussion is essential. This is based in parts on the currently used Schwartz Round. ${ }^{17}$ Predominantly used in hospitals and in wider medicine, discussion of the social and emotional impact of clinical aspects of patient care is integrated with the aim to reduce professional stress and anxiety. Diverting slightly from this model, which suggests all members (non-clinical and clinical) come together, each team member would have the opportunity to be assigned to another of similar role in order to satisfy peer group relationships. This pairing would occur over a period of three months, after which the team would come together as a whole group to discuss positive outcomes from each pairing and then a new permutation of pairings would be formed at random, and the cycle repeated. There is much to be said for not feeling alone when faced with a problem and a sense of relief in knowing fellow peers have experienced or are experiencing similar challenges, particularly with patient management.

Unfortunately, the challenge common to all aspects of modern health care: role ambiguity and constraint of time, prevents mentorship from being explicitly active..$^{18} \mathrm{~A}$ lack of boundaries often means that mentors take on the problems of mentees beyond their capability, leading to a counterproductive setup. There is also a strong argument for providing mentors with adequate training so that specific skills are taught, such as time management, defining roles, and managing expectations. In general dental practice, the question then arises as to who would provide such training. Presumably, training would need to be sought external to the practice, and then one would ask, who would give up their time solely to mentor others without an incentive. Similar to servant leadership, it appears the concept of 'helping power motivation' emerges in mentoring also, but it is not easy to find many who possess such motivation.

\section{Conclusion}

The common characteristics to enhance team morale are to encourage autonomy among team members in the following ways: a) by changing leadership strategy to one that is conducive to promoting empowerment; $b$ ) introducing a buddy/mentorship programme which also focuses on individual development. Both strategies aim to form an ethos of psychological wellbeing by ensuring the focus of being in a team is not only to enhance patient care but also to motivate each team member.

The focus of these strategies is intended to strengthen the team through placing value in each team member. These strategies have their merits and their challenges, the biggest challenge remains seeking and training individuals who are motivated solely by helping others. Setting aside allocated and undivided time to train such individuals, and generating authentic trust within the team, is equally challenging. The dynamic of trust, once established, is also likely to become disrupted once a new member of staff joins or another leaves and, therefore, the strategy relies on a stable servant leader to maintain the ethos of the team.

There is enough evidence to support the need for 'rehumanising' teams in health care, and especially in dental practice. Health care can be physically demanding, but most notably mentally and emotionally challenging. There is no doubt that a reduction in mental wellbeing of staff has a direct negative impact on patient care and, therefore, an investment into improving staff wellbeing is paramount. Put simply, if no one cares about each member of the dental team, why should they care about patients?

\section{References}

1. Wickstrom G, Bendix T. The "Hawthorne Effect" - what did the original Hawthorne studies actually show? Scand J Work Environ Health 2002; 26: 363-367.

2. Thomas C H, Lankau M J. Preventing burnout: the effects of LMX and mentoring on socialization, role stress, and burnout. Hum Resour Manage 2009; 48: 417-432.

3. Royal College of Physicians. Work and wellbeing in the NHS: why staff health matters to patient care. 2015. Available at https://www.rcplondon.ac.uk/guidelinespolicy/work-and-wellbeing-nhs-why-staff-healthmatters-patient-care (accessed March 2019).

4. Tonic. Are Dentists Really More Prone to Suicide? 2017. Available at https://tonic.vice.com/amp/en_us/ article $/ 5344$ jz/are $\% 20$ dentists $\% 20$ really $\% 20$ more $\% 20$ prone $\% 20$ to\%20suicide (accessed March 2019).

5. Finlayson B. Counting the Smiles: Morale and motivation in the NHS. 2002. Available at https://www.kingsfund.org.uk/ publications/counting-smiles (accessed March 2019).

6. Maben J, Peccei R, Adams M et al. Exploring the relationship between patients' experiences of care and the influence of staff motivation, affect and wellbeing. 2012. Available at http://www.netscc.ac.uk/hsdr/files/project/ SDO_ES_08-1819-213_V01.pdf (accessed March 2019).

7. Al Hassan A. Defensive dentistry and the young dentist - this isn't what we signed up for. Br Dent J 2017; 223: 757-758.

8. Lee C, Scheunemann J, Hall R, Payne L. Low Staff Morale \& Burnout: Causes \& Solutions. 2012. Available at https://lb.webservices.illinois.edu files/2012/06/01/39974.pdf (accessed March 2019).

9. Greenleaf R K (ed). Servant Leadership: A Journey into the Nature of Legitimate Power and Greatness. Mahwah NJ: Paulist Press, 2002.

10. Trastek V F, Hamilton N W, Niles E E. Leadership models in health care: a case for servant leadership. Mayo Clin Proc 2014; 89: 374-381.

11. Hanse J J, Harlin U, Jarebrant C, Ulin K, Winkel J. The impact of servant leadership dimensions on leadermember exchange among health care professionals. J Nurs Manag 2015; 24: 228-234.

12. Brown S, Bryant P. Getting to Know the Elephant: A Call to Advance Servant Leadership through Construct Consensus, Empirical Evidence, and Multilevel Theoretical Development. Serv Leader 2015; 2: 10-35.

13. Lee $P$, Gillespie $N$, Mann L, Wearing A. Leadership and trust: Their effect on knowledge sharing and team performance. Manag Learn 2010; 41: 473-491.

14. Stone A G, Russell R F, Patterson K. Transformational versus servant leadership: a difference in leader focus. Leadership Org Dev J 2004; 25: 349-361.

15. van Dierendonck D. Servant Leadership: A Review and Synthesis. J Manag 2011; 37: 1228-1261.

16. Holt V P, Ladwa R. Developing a mentoring culture in dentistry. Making a difference in a changing world. Prim Dent Care 2002; 17: 93-98.

17. Royal College of Surgeons. The morale maze: what's to be done to improve workforce morale? 2016. Available at https://www.rcseng.ac.uk/news-and-events/blog/ improving-workforce-morale/ (accessed March 2019).

18. MacLeod $\mathrm{S}$. The challenge of providing mentorship in primary care. Postgrad Med J 2007; 83: 317-319. 